\title{
Maximizing the Efficiency of Atomic and Molecular Imaging Using SIMS
}

\author{
Peter Williams \\ Department of Chemistry \& Biochemistry, Arizona State University, Tempe, AZ 85287
}

\begin{abstract}
SIMS analysis today offers the ability to perform chemical microscopy with spatial resolution up to an order of magnitude better than the diffraction limit of optical microscopy, with the additional possibilities of isotopic and molecular identification. The resolution limit is imposed partly by the size of the focused primary ion beam, and partly by the fact that there are very few atoms or molecules in the sampled volume at the smallest beam sizes. Thus it becomes extremely important to be able to sample the target material with the highest possible efficiency. Mass spectrometer design has now advanced to the point where ion transmission and detection with close to $100 \%$ efficiency is possible. The limiting factor then becomes the efficiency with which we can produce ions from the sampled species.
\end{abstract}

Cesium and oxygen primary ion beams have long been used to increase the ionization efficiency of negative and positive ions respectively, and for inorganic materials their ionization-enhancing effects are at least qualitatively understood in terms of how they alter the surface electronic structure and influence electron exchange between the surface and the departing sputtered particles. However, biological material is an increasingly frequent target and there is no good picture yet of what the electronic structure of such surfaces might be, or how this might be altered by high-dose implantation of these reactive primary ions. In this talk I will outline what is known about secondary ion emission processes and try to extrapolate to ionization issues in biological materials, both for molecular and for atomic ion emission. 